\title{
Finite-dimensional Lie subalgebras of algebras with continuous inversion
}

\author{
by \\ Daniel Beltiţă (Bucureşti) and \\ Karl-Hermann NeEb (Darmstadt)
}

\begin{abstract}
We investigate the finite-dimensional Lie groups whose points are separated by the continuous homomorphisms into groups of invertible elements of locally convex algebras with continuous inversion that satisfy an appropriate completeness condition. We find that these are precisely the linear Lie groups, that is, the Lie groups which can be faithfully represented as matrix groups. Our method relies on proving that certain finite-dimensional Lie subalgebras of algebras with continuous inversion commute modulo the Jacobson radical.
\end{abstract}

1. Introduction. In the present paper we investigate the finite-dimensional Lie groups whose points are separated by the continuous homomorphisms into groups of invertible elements of locally convex algebras with continuous inversion. We find that these are precisely the linear Lie groups, that is, the Lie groups which can be faithfully represented as matrix groups (see Theorem 5.3 below). The structure of the linear groups is fairly well understood; see for instance Hochschild's book [Ho65].

This problem is motivated by recent developments in the Lie theory of infinite-dimensional Lie groups modeled on locally convex spaces (cf. [Mil83], [Bel06], [GN06]). In this context, the unit groups of continuous inverse algebras are the prototypical "linear Lie groups" ([G102]), and it is a natural question whether the notion of "linearity" in this general context determines a larger class of finite-dimensional Lie groups than the Lie groups of matrices.

The unital Banach algebras provide examples of locally convex algebras with continuous inversion, and in this special case we recover the

2000 Mathematics Subject Classification: Primary 22E15; Secondary 22E65, 46H30, $17 \mathrm{~B} 30$.

Key words and phrases: linear Lie group, faithful representation, algebra with continuous inversion. 
characterization obtained in the paper [LV94] for the Lie groups whose uniformly continuous representations separate points. However, it is not clear to us if the approach used in [LV94] can be extended to non-normable algebras. For one thing, one of the key tools used in the aforementioned paper was a version of Lie's theorem concerning existence of weights for infinite-dimensional representations of solvable Lie algebras. Such a version is available as yet only for representations by Banach space operators (see [GL73] and [BS01]). It is worth mentioning at this point that there exist large classes of algebras with continuous inversion which are not Banach algebras, for instance algebras of germs of holomorphic functions or algebras of smooth matrix-valued functions on compact manifolds; see Examples VIII.3 in [Ne06] for more details and additional examples.

Thus an alternative approach is needed when working with general locally convex algebras with continuous inversion. For this purpose we prove that the main result of [Ti87] actually holds true far beyond the setting of Banach algebras, where it was originally discovered. Loosely speaking, we show that if $\mathfrak{g}$ is a finite-dimensional complex solvable Lie subalgebra of an algebra with continuous inversion that satisfies an appropriate completeness condition, then the closed unital associative subalgebra generated by $\mathfrak{g}$ is commutative modulo its (Jacobson) radical; see Theorem 4.3 below, which is the main technical result we need here. We obtain it by a method inspired from Turovski1's proof in the version presented in [BS01]. However, we emphasize that the present paper can be read independently of that book, inasmuch as several of the key tools developed in [BS01] - notably the Kleinecke-Shirokov theorem and the spectral theory for several non-commuting variables - are not readily available beyond the setting of normable algebras, so that we now replace them by arguments of a different type. And we strive to take advantage of this situation in order to make the present paper fairly selfcontained.

In Section 2 we set up the necessary preliminaries on algebras with continuous inversion and on nilpotent elements in Lie subalgebras of associative algebras. Section 3 concerns spectra of commutators, and includes in particular a version of the Kleinecke-Shirokov theorem holding for not necessarily normable algebras, as well as a version of Rosenblum's theorem from [Ro56] suitable for our purposes. In Section 4 we prove the theorem on commutativity modulo the radical (Theorem 4.3).

Finally, in Section 5 we obtain the main result of the present paper, namely the characterization of the finite-dimensional Lie groups whose points are separated by the homomorphisms into groups of invertible elements of FC-complete algebras with continuous inversion. 


\section{Preliminaries}

Preliminaries on algebras with continuous inversion

Notation 2.1. For an arbitrary unital complex associative algebra $\mathcal{A}$ we shall use the following notation:

- $\mathcal{A}^{\times}=\left\{a \in \mathcal{A} \mid\left(\exists a^{-1} \in \mathcal{A}\right) a a^{-1}=a^{-1} a=\mathbf{1}\right\}$;

- the spectrum of any $a \in \mathcal{A}$ is $\sigma_{\mathcal{A}}(a)=\sigma(a)=\left\{\lambda \in \mathbb{C} \mid \lambda \mathbf{1}-a \notin \mathcal{A}^{\times}\right\}$;

- the spectral radius of any $a \in \mathcal{A}$ is $r_{\mathcal{A}}(a)=\sup \{|\lambda| \mid \lambda \in \sigma(a)\} \in$ $[0, \infty]$;

- the center $\mathcal{Z}_{\mathcal{A}}=\{a \in \mathcal{A} \mid(\forall b \in \mathcal{A}) a b=b a\}$;

- $\mathcal{N}_{\mathcal{A}}=\left\{a \in \mathcal{A} \mid(\exists N \in \mathbb{N}) a^{N}=0\right\}$;

- $\mathcal{Q}_{\mathcal{A}}=\{a \in \mathcal{A} \mid \sigma(a)=\{0\}\}=\left\{a \in \mathcal{A} \mid \mathbf{1}+\mathbb{C} a \subseteq \mathcal{A}^{\times}\right\}$;

- the radical $\operatorname{rad} \mathcal{A}=\left\{a \in \mathcal{A} \mid(\forall b \in \mathcal{A}) \mathbf{1}-a b \in \mathcal{A}^{\times}\right\} \subseteq \mathcal{Q}_{\mathcal{A}}$.

Moreover, for any complex vector space $\mathcal{X}$ we denote by $\mathcal{L}(\mathcal{X})$ the set of all linear maps from $\mathcal{X}$ into itself.

Definition 2.2. A continuous inverse algebra (CIA for short) is a Hausdorff locally convex unital algebra $\mathcal{A}$ whose unit group $\mathcal{A}^{\times}$is open and for which the inversion map $\mathcal{A}^{\times} \rightarrow \mathcal{A}, a \mapsto a^{-1}$, is continuous.

For any element $a$ of a complex CIA $\mathcal{A}$ the spectrum $\sigma(a)$ is a non-empty compact subset of $\mathbb{C}$. Here the boundedness of the spectrum follows from

$$
r_{\mathcal{A}}(a)=\left(\sup \left\{r>0|| z \mid \leq r \Rightarrow \mathbf{1}+z a \in \mathcal{A}^{\times}\right\}\right)^{-1}
$$

and its closedness from the openness of the unit group $\mathcal{A}^{\times}$.

If, in addition, $\mathcal{A}$ is complete, then the same arguments as for Banach algebras lead to a holomorphic functional calculus ([Wae67], [G102]). Since completeness is in general not inherited by quotients $([$ Koe69, $\S 31.6])$, it is natural to consider for CIAs the weaker condition of FC-completeness, that is, closedness under holomorphic functional calculus. This means that for $a \in A$, any open neighborhood $U$ of $\sigma(a)$, each holomorphic function $f \in \mathcal{O}(U)$ and any contour $\Gamma$ around $\sigma(a)$ in $U$, the integral

$$
f(a):=\frac{1}{2 \pi i} \oint_{\Gamma} f(\zeta)(a-\zeta \mathbf{1})^{-1} d \zeta,
$$

which defines an element of the completion of $\mathcal{A}$, actually exists in $\mathcal{A}$.

REMARK 2.3. It is known that in every unital complex associative algebra $\mathcal{A}$ the radical $\operatorname{rad} \mathcal{A}$ is equal to the intersection of all the maximal left ideals of $\mathcal{A}$, and it is equal to the intersection of all the maximal right ideals of $\mathcal{A}$ as well. (See for instance Theorem 3.53 in Chapter 1 of [He93].) In particular, $\operatorname{rad} \mathcal{A}$ is a two-sided ideal of $\mathcal{A}$, and we have $\operatorname{rad}(\mathcal{A} / \operatorname{rad} \mathcal{A})=\{0\}$. (See, e.g., Theorem 3.63 in Chapter 1 of [He93].) 
If $\mathcal{A}$ is a CIA, then the fact that its unit group is open implies that every maximal left (or right) ideal of $\mathcal{A}$ is closed (see, e.g., Subsection 2.3 in Chapter II of [Wae67], or Corollary 3.9 in Chapter 2 of [Co68]), hence $\operatorname{rad} \mathcal{A}$ is a closed two-sided ideal of $\mathcal{A}$. Then the quotient algebra $\mathcal{A} / \operatorname{rad} \mathcal{A}$ is in turn a locally convex unital algebra with continuous inversion. (See, e.g., Subsection 2.2 in Chapter II of [Wae67] on quotients by closed ideals, or Prop. 3.14 in Chapter 2 of [Co68].)

Lemma 2.4. If $\mathcal{A}$ is a commutative complex CIA, then the spectral radius $r_{\mathcal{A}}$ is a continuous submultiplicative seminorm and, in particular, $\operatorname{rad} \mathcal{A}$ $=\mathcal{Q}_{\mathcal{A}}$.

Proof (cf. [Bi07, Th. 1.7]). The spectrum $\widehat{\mathcal{A}}:=\operatorname{Hom}(\mathcal{A}, \mathbb{C})$ of $\mathcal{A}$ is a compact Hausdorff space and the Gelfand transform $\mathcal{G}: \mathcal{A} \rightarrow C(\widehat{A}), a \mapsto$ $\widehat{a}, \widehat{a}(\chi):=\chi(a)$, is a continuous homomorphism of complex unital locally convex algebras, satisfying $\widehat{a}(\widehat{\mathcal{A}})=\sigma(a)$ and hence $\|\widehat{a}\|_{\infty}=r_{\mathcal{A}}(a)$. It follows that for each quasi-nilpotent element $a \in \mathcal{A}$ and $b \in \mathcal{A}$ we have $r_{\mathcal{A}}(a b) \leq$ $r_{\mathcal{A}}(a) r_{\mathcal{A}}(b)=0$, so that $\mathbf{1}-a b \in \mathcal{A}^{\times}$, so $a \in \operatorname{rad} \mathcal{A}$. This implies that $\operatorname{rad} \mathcal{A} \subseteq \mathcal{Q}_{\mathcal{A}} \subseteq \operatorname{rad} \mathcal{A}$, proving the asserted equality.

An early reference for our Lemma 2.4 is for instance [Wae67]. Another proof follows from Lemma II.9, Proposition II.3, and Corollary III.9 in [KOO98].

Lemma 2.5. Let $\mathcal{A}$ be a complex CIA.

(1) Each closed unital subalgebra $\mathcal{S}$ of $\mathcal{A}$ is a CIA. If $\mathcal{A}$ is FC-complete, then so is $\mathcal{S}$.

(2) If $\mathcal{S} \leq \mathcal{A}$ is a maximal commutative subalgebra, then $\mathcal{S}$ is unital, closed and equispectral, i.e., $\sigma_{\mathcal{A}}(x)=\sigma_{\mathcal{S}}(x)$ for each $x \in \mathcal{S}$.

(3) For each closed ideal $\mathcal{I} \unlhd \mathcal{A}$ the quotient algebra $\mathcal{Q}:=\mathcal{A} / \mathcal{I}$ is a CIA. If , in addition, $\mathcal{A}$ is $F C$-complete, and if the quotient map $q: \mathcal{A} \rightarrow \mathcal{Q}$ satisfies $\sigma_{\mathcal{A}}(a)=\sigma_{\mathcal{Q}}(q(a))$ for each $a \in A$, then $\mathcal{Q}$ is also FC-complete.

Proof. (1) (See also Remarque 4.6 and Subsection 4.3 in Chapter 2 of [Wae67].) If $a \in \mathcal{S}$ satisfies $r_{\mathcal{A}}(a)<1$, then $\mathbf{1}-a$ is invertible and the Neumann series $\sum_{n=0}^{\infty} a^{n}$ converges to $(\mathbf{1}-a)^{-1}$ ([G102]). Since $\mathcal{S}$ is closed, $(\mathbf{1}-a)^{-1} \in \mathcal{S}$, so that $\mathcal{S}^{\times}$is a neighborhood of $\mathbf{1}$ in $\mathcal{S}$, hence open. The continuity of the inversion in $\mathcal{S}$ follows from the corresponding property of $\mathcal{A}$.

If, in addition, $\mathcal{A}$ is FC-complete and $s \in \mathcal{S}$, then $\sigma_{\mathcal{S}}(s) \supseteq \sigma_{\mathcal{A}}(s)$, so that each contour around $\sigma_{\mathcal{S}}(s)$ also surrounds $\sigma_{\mathcal{A}}(s)$. Now for any holomorphic 
function $f$ on a neighborhood of $\sigma_{\mathcal{S}}(s)$ the integral

$$
f(s):=\frac{1}{2 \pi i} \oint_{\Gamma} f(\zeta)(s-\zeta \mathbf{1})^{-1} d \zeta
$$

defines an element of $\mathcal{A}$. Since $(s-\zeta \mathbf{1})^{-1} \in \mathcal{S}$ for each $\zeta$, the closedness of $\mathcal{S}$ yields $f(s) \in \mathcal{S}$. This shows that $\mathcal{S}$ is FC-complete.

(2) Since closures of commutative subalgebras are commutative subalgebras, the maximality of $\mathcal{S}$ implies its closedness. This trivially implies $\mathbf{1} \in \mathcal{S}$. Moreover, for each $x \in \mathcal{S}$ we have $(x-\lambda \mathbf{1})^{-1} \in \mathcal{S}$ whenever $x-\lambda \mathbf{1}$ is invertible in $\mathcal{A}$ because $(x-\lambda \mathbf{1})^{-1}$ commutes with all elements of $\mathcal{S}$. This leads to $r_{\mathcal{A}}(x)=r_{\mathcal{S}}(x)$.

(3) Since $q\left(\mathcal{A}^{\times}\right) \subseteq \mathcal{Q}^{\times}$is an open subset of $\mathcal{Q}$, the unit group $\mathcal{Q}^{\times}$is open. The continuity of the inversion of $\mathcal{Q}$ follows from its continuity at $\mathbf{1}$ and the continuity of the map $\mathcal{A}^{\times} \rightarrow \mathcal{Q}^{\times}, a \mapsto q(a)^{-1}=q\left(a^{-1}\right)$, because $q$ is an open map.

For each $a \in A$ we have $\sigma_{\mathcal{A}}(a)=\sigma_{\mathcal{Q}}(q(a))$. For any open neighborhood of $\sigma_{\mathcal{Q}}(q(a))$, each holomorphic function $f \in \mathcal{O}(U)$, and any contour around $\sigma(a)$ in $U$ the FC-completeness implies that the integral

$$
f(a):=\frac{1}{2 \pi i} \oint_{\Gamma} f(\zeta)(a-\zeta \mathbf{1})^{-1} d \zeta
$$

exists in $\mathcal{A}$. We conclude that the integral

$$
q(f(a))=\frac{1}{2 \pi i} \oint_{\Gamma} f(\zeta) q(a-\zeta \mathbf{1})^{-1} d \zeta=\frac{1}{2 \pi i} \oint_{\Gamma} f(\zeta)(q(a)-\zeta \mathbf{1})^{-1} d \zeta=f(q(a))
$$

exists in $\mathcal{Q}$. Hence $\mathcal{Q}$ is FC-complete.

Algebraic preliminaries. In this subsection we turn to the purely algebraic part of the preliminaries we need for our main results. In particular, we prove a suitable generalization of Theorem 2 in $\S 28$ of [BS01].

Proposition 2.6. Let $\mathcal{X}$ be a complex vector space, $\mathfrak{g}$ a finite-dimensional solvable Lie subalgebra of $\mathcal{L}(\mathcal{X})$, and $\mathfrak{h}$ a Cartan subalgebra of $\mathfrak{g}$. Denote by $\mathfrak{g}^{\alpha}, \alpha \in R$, the family of root spaces of $\mathfrak{g}$ corresponding to the set $R$ of non-zero roots, and assume that $\mathfrak{g}^{\alpha}$ consists of nilpotent elements for every $\alpha \in R$. Then $\mathcal{N}_{\mathcal{L}(\mathcal{X})} \cap \mathfrak{g}$ is an ideal of $\mathfrak{g}$.

Proof. The proof has two steps.

STEP 1. If $\mathfrak{g}$ is a nilpotent Lie algebra then the desired conclusion follows by precisely the same reasoning as in Step 1 of the proof of Theorem 2 in $\S 28$ of [BS01]. Specifically, we shall argue by induction on dim $\mathfrak{g}$. The assertion is clear if $\operatorname{dim} \mathfrak{g}=1$.

Now assume that $\operatorname{dim} \mathfrak{g}>1$ and $\mathcal{N}_{\mathcal{L}(\mathcal{X})} \cap \mathfrak{g} \neq\{0\}$. Since nilpotent elements are always polynomially central (Definition 2 in $\S 16$ of [BS01]), it 
follows by Theorem 1 in $\S 18$ of [BS01], applied with $I=\mathfrak{g}$ (see also [Sa96]), that there exists $Y \in \mathcal{N}_{\mathcal{L}(\mathcal{X})} \cap \mathfrak{g}$ such that $Y \neq 0$ and $[Y, T]=0$ for all $T \in \mathfrak{g}$. Let $m \geq 1$ with $Y^{m-1} \neq 0=Y^{m}$, and define $\mathcal{X}_{k}=\operatorname{Ker}\left(Y^{k}\right)$ for $k=0, \ldots, m$. Since $Y$ belongs to the center of $\mathfrak{g}$, it follows that $\{0\}=\mathcal{X}_{0} \subseteq \cdots \subseteq \mathcal{X}_{m}=\mathcal{X}$ is a nest of invariant subspaces for $\mathfrak{g}$. In particular, there exist representations

$$
\begin{aligned}
& \varrho_{k}: \mathfrak{g} \rightarrow \mathcal{L}\left(\mathcal{X}_{k} / \mathcal{X}_{k-1}\right), \\
& \varrho_{k}(T)\left(x+\mathcal{X}_{k-1}\right)=T x+\mathcal{X}_{k-1} \quad \text { for all } T \in \mathfrak{g} \text { and } x \in \mathcal{X}_{k},
\end{aligned}
$$

for $k=0, \ldots, m$. It is easy to see that

$$
\mathcal{N}_{\mathcal{L}(\mathcal{X})} \cap \mathfrak{g}=\bigcap_{k=0}^{n} \varrho_{k}^{-1}\left(\mathcal{N}_{\mathcal{L}\left(\mathcal{X}_{k} / \mathcal{X}_{k-1}\right)}\right)=\bigcap_{k=0}^{n} \varrho_{k}^{-1}\left(\mathcal{N}_{\mathcal{L}\left(\mathcal{X}_{k} / \mathcal{X}_{k-1}\right)} \cap \varrho_{k}(\mathfrak{g})\right),
$$

that is, $T \in \mathfrak{g}$ is nilpotent if and only if each $\varrho_{k}(T) \in \mathcal{L}\left(\mathcal{X}_{k} / \mathcal{X}_{k-1}\right)$ is nilpotent for $k=0, \ldots, m$.

On the other hand, $0 \neq Y \in \bigcap_{k=0}^{m}$ Ker $\varrho_{k}$, hence for each $k$ we have $\operatorname{dim} \varrho_{k}(\mathfrak{g})<\operatorname{dim} \mathfrak{g}$. Then the induction hypothesis shows $\mathcal{N}_{\mathcal{L}\left(\mathcal{X}_{k} / \mathcal{X}_{k-1}\right)} \varrho_{k}(\mathfrak{g})$ is an ideal of $\varrho_{k}(\mathfrak{g})$. Now (1) implies that $\mathcal{N}_{\mathcal{L}(\mathcal{X})} \cap \mathfrak{g}$ is an ideal of $\mathfrak{g}$ since pull-backs and intersections of ideals are again ideals.

SteP 2. We now proceed with the proof in the general case. Define $\mathfrak{g}_{+}:=\bigoplus_{\alpha \in R} \mathfrak{g}^{\alpha}$ so that the generalized root space decomposition of $\mathfrak{g}$ with respect to the Cartan subalgebra $\mathfrak{h}$ leads to the Fitting decomposition $\mathfrak{g}=$ $\mathfrak{h} \dot{+} \mathfrak{g}_{+}$. Next set $\mathfrak{g}_{0}=\left\{T \in \mathfrak{g} \mid \operatorname{ad}_{\mathfrak{g}} T: \mathfrak{g} \rightarrow \mathfrak{g}\right.$ is nilpotent $\}$. Since $\mathfrak{g}$ is a solvable Lie algebra, it follows easily from the Lie theorem on simultaneous triangularization of solvable Lie algebras of matrices that $\mathfrak{g}_{0}$ is an ideal of $\mathfrak{g}$. Moreover, $\mathfrak{g}_{0}$ is a nilpotent Lie algebra and

$$
\bigcup_{\alpha \in R} \mathfrak{g}^{\alpha} \subseteq \mathcal{N}_{\mathcal{L}(\mathcal{X})} \cap \mathfrak{g} \subseteq \mathfrak{g}_{0} .
$$

In particular, Step 1 of the proof shows that $\mathcal{N}_{\mathcal{L}(\mathcal{X})} \cap \mathfrak{g}$ is an ideal of $\mathfrak{g}_{0}$. To prove that $\mathcal{N}_{\mathcal{L}(\mathcal{X})} \cap \mathfrak{g}$ is an ideal of $\mathfrak{g}$ it remains to check that $\left[\mathfrak{h}, \mathcal{N}_{\mathcal{L}(\mathcal{X})} \cap \mathfrak{g}\right] \subseteq$ $\mathcal{N}_{\mathcal{L}(\mathcal{X})} \cap \mathfrak{g}$.

To this end note that since we have the inclusion of vector subspaces $\mathfrak{g}_{+} \subseteq \mathcal{N}_{\mathcal{L}(\mathcal{X})} \cap \mathfrak{g}$ and $\mathfrak{g}=\mathfrak{h} \dot{+} \mathfrak{g}_{+}$it follows that $\mathcal{N}_{\mathcal{L}(\mathcal{X})} \cap \mathfrak{g}=\left(\mathcal{N}_{\mathcal{L}(\mathcal{X})} \cap \mathfrak{h}\right) \dot{+} \mathfrak{g}_{+}$. Since $\mathfrak{g}=\mathfrak{h} \dot{+} \mathfrak{g}_{+}$it then follows that

$$
\left[\mathfrak{h}, \mathcal{N}_{\mathcal{L}(\mathcal{X})} \cap \mathfrak{g}\right] \subseteq\left[\mathfrak{h}, \mathcal{N}_{\mathcal{L}(\mathcal{X})} \cap \mathfrak{h}\right]+\left[\mathfrak{h}, \mathfrak{g}_{+}\right] .
$$

Now $\left[\mathfrak{h}, \mathcal{N}_{\mathcal{L}(\mathcal{X})} \cap \mathfrak{h}\right] \subseteq \mathcal{N}_{\mathcal{L}(\mathcal{X})} \cap \mathfrak{h}$ by Step 1 of the proof, since $\mathfrak{h}$ is a nilpotent Lie algebra. Moreover, $\left[\mathfrak{h}, \mathfrak{g}_{+}\right] \subseteq \mathfrak{g}_{+} \subseteq \mathcal{N}_{\mathcal{L}(\mathcal{X})} \cap \mathfrak{g}$, where the latter inclusion follows by the hypothesis since we saw that $\mathcal{N}_{\mathcal{L}(\mathcal{X})} \cap \mathfrak{g}$ is a vector space. Thus (2) shows that $\left[\mathfrak{g}, \mathcal{N}_{\mathcal{L}(\mathcal{X})} \cap \mathfrak{g}\right] \subseteq \mathcal{N}_{\mathcal{L}(\mathcal{X})} \cap \mathfrak{g}$, and the proof is complete. 
Lemma 2.7. Assume that $\mathcal{A}$ is a complex unital associative algebra and $\mathfrak{g}$ a finite-dimensional Lie subalgebra of $\mathcal{A}$ such that $\mathfrak{g} \subseteq \mathcal{N}_{\mathcal{A}}$. Then there exists an integer $m \geq 1$ such that $a_{1} \cdots a_{m}=0$ for all $a_{1}, \ldots, a_{m} \in \mathfrak{g}$.

Proof. Since ad $a$ is a nilpotent operator on $\mathcal{A}$ for each $a \in \mathfrak{g}$, Engel's theorem implies that $\mathfrak{g}$ is a nilpotent Lie algebra. In view of the PoincaréBirkhoff-Witt theorem, the unital associative subalgebra $\mathcal{A}(\mathfrak{g})$ generated by $\mathfrak{g}$ is finite-dimensional. Then Lie's theorem, applied to the left regular representation of $\mathfrak{g}$ on $\mathcal{A}(\mathfrak{g})$ implies that the associative subalgebra of $\mathcal{A}$ generated by $\mathfrak{g}$ consists of nilpotent elements.

\section{Spectra of commutators}

Proposition 3.1. Let $\mathcal{A}$ be a complex FC-complete CIA. Assume that $a, b, c \in \mathcal{A}$ satisfy $[a, b]=a b-b a=c, a c=c a$, and $b c=c b$. Then

$$
\sigma_{\mathcal{A}}(c)=\{0\} .
$$

Proof. Since the algebra $\mathcal{A}$ is FC-complete, it has an exponential function exp: $\mathcal{A} \rightarrow \mathcal{A}^{\times}, x \mapsto e^{x}$, defined by the holomorphic functional calculus. Define

$$
f: \mathbb{C} \rightarrow \mathcal{A}, \quad f(t)=e^{t a} b e^{-t a} .
$$

Then $f$ is holomorphic, $f(0)=b$ and $f^{\prime}(t)=a e^{t a} b e^{-t a}-e^{t a} b a e^{-t a}=$ $e^{t a} c e^{-t a}=c$ for all $t \in \mathbb{C}$, because of the assumption $a c=c a$. This implies that $e^{t a} b e^{-t a}=b+t c$ for each $t \in \mathbb{C}$, and hence

$$
\sigma_{\mathcal{A}}(b)=\sigma_{\mathcal{A}}(b+t c) \quad \text { for each } t \in \mathbb{C} .
$$

As $b$ and $c$ commute,

$$
|t| \sigma(c)=\sigma(t c)=\sigma(t c+b-b) \subseteq \sigma(t c+b)-\sigma(b)=\sigma(b)-\sigma(b) .
$$

Since $\sigma(b)$ is bounded, for $|t| \rightarrow \infty$ we obtain the inclusion $\sigma(c) \subseteq\{0\}$. The lemma now follows from the non-emptieness of the spectrum.

Lemma 3.2. For each complex FC-complete CIA $\mathcal{A}$ we have

$$
\mathcal{Z}_{\mathcal{A}} \cap \mathcal{Q}_{\mathcal{A}} \subseteq \operatorname{rad} \mathcal{A} .
$$

Proof. Let $c \in \mathcal{Z}_{\mathcal{A}} \cap \mathcal{Q}_{\mathcal{A}}$ and $b \in \mathcal{A}$. We have to show that $\mathbf{1}-b c \in \mathcal{A}^{\times}$. To this end, let $\mathcal{A}_{0}$ be a maximal commutative subalgebra of $\mathcal{A}$ containing both $b$ and $c$. Then $\mathcal{A}_{0}$ is a closed unital subalgebra of $\mathcal{A}$, hence an FCcomplete CIA with $r_{\mathcal{A}}(a)=r_{\mathcal{A}_{0}}(a)$ (Lemma 2.5). In particular, $r_{\mathcal{A}}(b c)=$ $r_{\mathcal{A}_{0}}(b c) \leq r_{\mathcal{A}_{0}}(b) r_{\mathcal{A}_{0}}(c)=0$, where the inequality follows by Lemma 2.4 . Thus $\sigma_{\mathcal{A}}(b c)=\{0\}$, and so $\mathbf{1}-b c \in \mathcal{A}^{\times}$, as desired.

Proposition 3.3. Let $\mathcal{A}$ be a complex FC-complete CIA. Then for all $a_{1}, a_{2} \in \mathcal{A}$ the operator

$$
\Delta: \mathcal{A} \rightarrow \mathcal{A}, \quad x \mapsto a_{1} x-x a_{2},
$$

satisfies $\sigma_{\mathcal{L}(\mathcal{A})}(\Delta) \subseteq \sigma_{\mathcal{A}}\left(a_{1}\right)-\sigma_{\mathcal{A}}\left(a_{2}\right)$. 
Proof. The method of proof used in Section 3 of [Ro56] works in the present setting as well. Specifically, let $\lambda \in \mathbb{C}$ with $\lambda \notin \sigma_{\mathcal{A}}\left(a_{1}\right)-\sigma_{\mathcal{A}}\left(a_{2}\right)$. We are going to prove that $\lambda \notin \sigma_{\mathcal{L}(\mathcal{A})}(\Delta)$. Since both $\sigma_{\mathcal{A}}\left(a_{1}\right)$ and $\sigma_{\mathcal{A}}\left(a_{2}\right)$ are compact subsets of $\mathbb{C}$, there exist open subsets $U_{1}$ and $U_{2}$ of $\mathbb{C}$ such that $\sigma_{\mathcal{A}}\left(a_{j}\right) \subseteq U_{j}$ for $j=1,2$ and $\bar{U}_{1} \cap\left(\lambda+\bar{U}_{2}\right)=\emptyset$.

Now let $\Gamma \subseteq U_{2}$ be a piecewise smooth contour surrounding $\sigma_{\mathcal{A}}\left(a_{2}\right)$. Then for every $z \in \Gamma$ we have $\lambda+z \notin \sigma_{\mathcal{A}}\left(a_{1}\right)$, hence we can define a linear map from $\mathcal{A}$ into itself by

$$
T: \mathcal{A} \rightarrow \mathcal{A}, \quad T x=\frac{1}{2 \pi i} \oint_{\Gamma}\left((\lambda+z) \mathbf{1}-a_{1}\right)^{-1} x\left(z \mathbf{1}-a_{2}\right)^{-1} d z .
$$

We now have

$$
\begin{aligned}
T(\lambda \mathbf{1}-\Delta) x & =T\left(\lambda x-a_{1} x+x a_{2}\right) \\
& =\frac{1}{2 \pi i} \oint_{\Gamma}\left((\lambda+z \mathbf{1})-a_{1}\right)^{-1}\left(\lambda x-a_{1} x+x a_{2}\right)\left(z \mathbf{1}-a_{2}\right)^{-1} d z \\
& =\frac{1}{2 \pi i} \oint_{\Gamma}\left((\lambda+z \mathbf{1})-a_{1}\right)^{-1} \\
& =\frac{1}{2 \pi i} \oint_{\Gamma} x\left(z \mathbf{1}-a_{2}\right)^{-1} d z-\frac{1}{2 \pi i} \oint_{\Gamma}\left((\lambda+z \mathbf{1})-a_{1}\right)^{-1} x d z \\
& =x \frac{1}{2 \pi i} \oint_{\Gamma}\left(z \mathbf{1}-a_{2}\right)^{-1} d z-\frac{1}{2 \pi i} \oint_{\Gamma}\left((\lambda+z \mathbf{1})-a_{1}\right)^{-1} d z \cdot x \\
& =x \cdot \mathbf{1}-\mathbf{0} \cdot x=x
\end{aligned}
$$

and

$$
\begin{aligned}
(\lambda \mathbf{1}-\Delta) T x= & \lambda T x-a_{1} T x+T x a_{2} \\
= & \frac{1}{2 \pi i} \oint_{\Gamma}\left(\lambda \mathbf{1}-a_{1}\right)\left((\lambda+z \mathbf{1})-a_{1}\right)^{-1} x\left(z \mathbf{1}-a_{2}\right)^{-1} d z \\
& +\frac{1}{2 \pi i} \oint_{\Gamma}\left((\lambda+z \mathbf{1})-a_{1}\right)^{-1} x\left(z \mathbf{1}-a_{2}\right)^{-1} a_{2} d z \\
= & \frac{1}{2 \pi i} \oint_{\Gamma}\left((\lambda+z-z) \mathbf{1}-a_{1}\right)\left((\lambda+z \mathbf{1})-a_{1}\right)^{-1} x\left(z \mathbf{1}-a_{2}\right)^{-1} d z \\
& +\frac{1}{2 \pi i} \oint_{\Gamma}\left((\lambda+z \mathbf{1})-a_{1}\right)^{-1} x\left(z \mathbf{1}-a_{2}\right)^{-1}\left(a_{2}-z \mathbf{1}+z \mathbf{1}\right) d z \\
= & x \frac{1}{2 \pi i} \oint_{\Gamma}\left(z \mathbf{1}-a_{2}\right)^{-1} d z+\frac{1}{2 \pi i} \oint_{\Gamma}\left((\lambda+z \mathbf{1})-a_{1}\right)^{-1} d z \cdot x \\
= & x \cdot \mathbf{1}+\mathbf{0} \cdot x=x .
\end{aligned}
$$

This shows that $T$ is an inverse of $\lambda \mathbf{1}-\Delta$, so that $\lambda \in \mathbb{C} \backslash \sigma_{\mathcal{L}(\mathcal{A})}(\Delta)$. 
Corollary 3.4. Let $\mathcal{A}$ be a complex FC-complete CIA. Assume that $a, b \in \mathcal{A}$ satisfy the condition $\left(\operatorname{ad}_{\mathcal{A}} a-\lambda\right)^{m} b=0$ for some $\lambda \in \mathbb{C} \backslash\{0\}$ and $m \geq 1$, where $\operatorname{ad}_{\mathcal{A}} a: \mathcal{A} \rightarrow \mathcal{A},\left(\operatorname{ad}_{\mathcal{A}} a\right) x=a x-x a$. Then for every integer $N>2 r_{\mathcal{A}}(a) /|\lambda|$ we have $b^{N}=0$.

Proof. Since $\Delta:=\operatorname{ad}_{\mathcal{A}} a: \mathcal{A} \rightarrow \mathcal{A}$ is a derivation of $\mathcal{A}$, it follows by induction on $k$ that for all integers $n, k \geq 1$, all $\lambda_{1}, \ldots, \lambda_{k} \in \mathbb{C}$, and all $x_{1}, \ldots, x_{k} \in \mathcal{A}$ we have

$$
\begin{aligned}
\left(\Delta-\lambda_{1}-\cdots-\lambda_{k}\right)^{n}\left(x_{1} \cdots x_{k}\right) & \\
& =\sum_{j_{1}+\cdots+j_{k}=n} \frac{n !}{j_{1} ! \cdots j_{k} !}\left(\left(\Delta-\lambda_{1}\right)^{j_{1}} x_{1}\right) \cdots\left(\left(\Delta-\lambda_{k}\right)^{j_{k}} x_{k}\right) .
\end{aligned}
$$

Hence $(\Delta-k \lambda)^{n}\left(b^{k}\right)=0$ whenever $k \geq 1$ and $n>k m$.

This shows that if $N \geq 1$ and $b^{N} \neq 0$, then $N \lambda \in \sigma_{\mathcal{L}(\mathcal{A})}(\Delta)$. Consequently, $N \lambda \in \sigma_{\mathcal{A}}(a)-\sigma_{\mathcal{A}}(a)$ by Proposition 3.3, whence necessarily $N|\lambda| \leq 2 r_{\mathcal{A}}(a)$.

4. Commutativity modulo the radical. The following statement is a version of Lemma 1 in $\S 24$ of [BS01].

Lemma 4.1. Let $\mathcal{A}$ be a unital complex FC-complete CIA with zero radical. Assume that $\mathfrak{g}$ is a complex Lie subalgebra of $\mathcal{A}$ such that the closed unital associative subalgebra generated by $\mathfrak{g}$ is equal to $\mathcal{A}$, and let $\mathfrak{j}$ be a finite-dimensional ideal of $\mathfrak{g}$ such that $\left.\left(\operatorname{ad}_{\mathfrak{g}} a\right)\right|_{\mathfrak{j}}: \mathfrak{j} \rightarrow \mathfrak{j}$ is a nilpotent map for every $a \in \mathfrak{g}$. Then $[\mathfrak{g}, \mathfrak{j}]=\{0\}$.

Proof. We essentially follow the lines of the proof of Lemma 1 in $\S 24$ of [BS01], by using Proposition 3.1 instead of the Kleinecke-Shirokov theorem. Since $\operatorname{dim} \mathfrak{j}<\infty$, it follows from Lemma 2.7 that there exists some $m \geq 1$ such that

$$
\left(\forall a_{1}, \ldots, a_{m} \in \mathfrak{g}\right)\left(\forall a_{0} \in \mathfrak{j}\right) \quad\left(\operatorname{ad}_{\mathfrak{g}} a_{m}\right) \cdots\left(\operatorname{ad}_{\mathfrak{g}} a_{1}\right) a_{0}=0 .
$$

We shall prove that if $m \geq 2$ then (3) also holds with $m-1$ instead of $m$, and this will conclude the proof.

To this end, let $a_{1}, \ldots, a_{m-1} \in \mathfrak{g}$ and $a_{0} \in \mathfrak{j}$, and set

$$
y=\left(\operatorname{ad}_{\mathfrak{g}} a_{m-1}\right) \cdots\left(\operatorname{ad}_{\mathfrak{g}} a_{1}\right) a_{0} \in \mathfrak{j} .
$$

We have to check that $y=0$. By (3) we have

$$
\left[a_{m}, y\right]=\left(\operatorname{ad}_{\mathfrak{g}} a_{m}\right)\left(\operatorname{ad}_{\mathfrak{g}} a_{m-1}\right) \cdots\left(\operatorname{ad}_{\mathfrak{g}} a_{1}\right) a_{0}=0 \quad \text { for all } a_{m} \in \mathfrak{g} .
$$

Since $\mathcal{A}$ is generated by $\mathfrak{g}$ it then follows that $a y=y a$ for all $a \in \mathcal{A}$, that is, $y \in \mathcal{Z}_{\mathcal{A}}$.

On the other hand, note that

$$
y=\left[a_{m-1}, u\right], \quad \text { where } \quad u=\left(\operatorname{ad}_{\mathfrak{g}} a_{m-2}\right) \cdots\left(\operatorname{ad}_{\mathfrak{g}} a_{1}\right) a_{0}
$$


if $m \geq 3$ and $u=a_{0}$ if $m=2$. Since $y$ commutes with every element in $\mathcal{A}$, we have in particular $y a_{m-1}=a_{m-1} y$ and $y u=u y$, hence Proposition 3.1 shows that $\sigma(y)=\{0\}$. Consequently, by Lemma 3.2, we get $y \in \mathcal{Q}_{\mathcal{A}} \cap \mathcal{Z}_{\mathcal{A}} \subseteq$ $\operatorname{rad} \mathcal{A}=\{0\}$, and the proof ends.

Here is a suitable version of Proposition 1 in $\S 24$ of [BS01].

Proposition 4.2. Let $\mathcal{A}$ be a complex FC-complete CIA. Assume that $\mathfrak{g}$ is a complex Lie subalgebra of $\mathcal{A}$ such that the closed unital associative subalgebra generated by $\mathfrak{g}$ equals $\mathcal{A}$, and let $\mathfrak{j}$ be a finite-dimensional solvable ideal of $\mathfrak{g}$. Then $\mathcal{N}_{\mathcal{A}} \cap \mathfrak{j}$ is an ideal of $\mathfrak{g}$ and $\mathcal{N}_{\mathcal{A}} \cap \mathfrak{j} \subseteq \operatorname{rad} \mathcal{A}$.

Proof. We follow the lines of the proof of Proposition 1 in $\S 24$ of [BS01]. Thus, let $\varrho: \mathcal{A} \rightarrow \mathcal{L}(\mathcal{A})$ be the regular representation of $\mathcal{A}$. Then $\varrho(\mathfrak{g})$ is a Lie subalgebra of $\mathcal{L}(\mathcal{A})$ and $\varrho(\mathfrak{j})$ is a finite-dimensional solvable ideal of $\varrho(\mathfrak{g})$.

On the other hand, let $\mathfrak{h}$ be a Cartan subalgebra of $\mathfrak{j},\left(\mathfrak{j}^{\alpha}\right)_{\alpha \in R}$ the root spaces of $\mathfrak{j}$ corresponding to the set $R$ of non-zero roots, and $\mathfrak{j}=\mathfrak{h} \dot{+} \mathfrak{g}_{+}$the corresponding Fitting decomposition, as in Proposition 2.6 and its proof. It follows from Corollary 3.4 that for every root $\alpha \in R$ we have $\mathrm{j}^{\alpha} \subseteq \mathcal{N}_{\mathcal{A}}$, and hence $\varrho\left(\mathrm{j}^{\alpha}\right) \subseteq \mathcal{N}_{\mathcal{L}(\mathcal{A})}$. Now note that $\varrho: \mathcal{A} \rightarrow \mathcal{L}(\mathcal{A})$ is a faithful representation, hence $\varrho(\mathfrak{h})$ is a Cartan subalgebra of $\varrho(\mathfrak{j})$ and $\left(\varrho\left(\mathfrak{j}^{\alpha}\right)\right)_{\alpha \in R}$ are the corresponding root spaces. Also, $\varrho\left(\mathcal{N}_{\mathcal{A}}\right) \subseteq \mathcal{N}_{\mathcal{L}(\mathcal{A})}$. Thus, we can apply Proposition 2.6 to deduce that $\varrho\left(\mathcal{N}_{\mathcal{A}} \cap \mathbf{j}\right)$ is an ideal of $\varrho(\mathfrak{j})$. Since $\varrho$ is faithful, this shows that $\mathcal{N}_{\mathcal{A}} \cap \mathfrak{j}$ is an ideal of $\mathfrak{j}$.

Now, to prove that $\mathcal{N}_{\mathcal{A}} \cap \mathfrak{j}$ is even an ideal of $\mathfrak{g}$, it suffices to check that $\left[a, \mathcal{N}_{\mathcal{A}} \cap \mathfrak{j}\right] \subseteq \mathcal{N}_{\mathcal{A}}$ for all $a \in \mathfrak{g}$. To this end, set $\mathfrak{j}_{1}=\mathbb{C} a+\mathfrak{j}$, which is a finite-dimensional solvable Lie subalgebra of $\mathfrak{g}$ since $\mathfrak{j}$ is a finite-dimensional solvable ideal. Then the preceding argument shows that $\mathcal{N}_{\mathcal{A}} \cap \mathfrak{j}_{1}$ is an ideal of $\mathfrak{j}_{1}$. If it happens that $\mathcal{N}_{\mathcal{A}} \cap \mathfrak{j}_{1}=\mathcal{N}_{\mathcal{A}} \cap \mathfrak{j}$, then $\left[a, \mathcal{N}_{\mathcal{A}} \cap \mathfrak{j}\right]=\left[a, \mathcal{N}_{\mathcal{A}} \cap \mathfrak{j}_{1}\right] \subseteq$ $\mathcal{N}_{\mathcal{A}} \cap \mathfrak{j}_{1}=\mathcal{N}_{\mathcal{A}} \cap \mathbf{j}$ and we are done. Now assume that $\mathcal{N}_{\mathcal{A}} \cap \mathfrak{j}_{1} \neq \mathcal{N}_{\mathcal{A}} \cap \mathbf{j}$ and pick $b \in\left(\mathcal{N}_{\mathcal{A}} \cap \mathfrak{j}_{1}\right) \backslash \mathfrak{j}$. Then $\mathbf{j}_{1}=\mathbb{C} b+\mathfrak{j}=\left(\mathcal{N}_{\mathcal{A}} \cap \mathfrak{j}_{1}\right)+\mathfrak{j}$, hence

$$
\begin{aligned}
\operatorname{dim}\left(\left(\mathcal{N}_{\mathcal{A}} \cap \mathfrak{j}_{1}\right) /\left(\mathcal{N}_{\mathcal{A}} \cap \mathfrak{j}\right)\right) & =\operatorname{dim}\left(\left(\mathcal{N}_{\mathcal{A}} \cap \mathfrak{j}_{1}\right) /\left(\left(\mathcal{N}_{\mathcal{A}} \cap \mathfrak{j}_{1}\right) \cap \mathfrak{j}\right)\right) \\
& =\operatorname{dim}\left(\left(\left(\mathcal{N}_{\mathcal{A}} \cap \mathfrak{j}_{1}\right)+\mathfrak{j}\right) / \mathfrak{j}\right)=\operatorname{dim}\left(\mathfrak{j}_{1} / \mathfrak{j}\right) \leq 1 .
\end{aligned}
$$

Since $b \in\left(\mathcal{N}_{\mathcal{A}} \cap \mathfrak{j}_{1}\right) \backslash\left(\mathcal{N}_{\mathcal{A}} \cap \mathfrak{j}\right)$, it follows that $\mathcal{N}_{\mathcal{A}} \cap \mathfrak{j}_{1}=\mathbb{C} b+\left(\mathcal{N}_{\mathcal{A}} \cap \mathbf{j}\right)$. Now the finite-dimensional Lie algebra $\mathcal{N}_{\mathcal{A}} \cap j_{1}$ consists of nilpotent elements, hence it is nilpotent. As every hyperplane subalgebra of a finite-dimensional nilpotent Lie algebra is an ideal, $\mathcal{N}_{\mathcal{A}} \cap \mathbf{j}$ is an ideal of $\mathcal{N}_{\mathcal{A}} \cap \mathrm{j}_{1}$. Since $\mathcal{N}_{\mathcal{A}} \cap \mathbf{j}$ is an ideal of $\boldsymbol{j}$ it follows that

$$
\left[a, \mathcal{N}_{\mathcal{A}} \cap \mathbf{j}\right] \subseteq\left[\mathbf{j}+\left(\mathcal{N}_{\mathcal{A}} \cap \mathfrak{j}_{1}\right), \mathcal{N}_{\mathcal{A}} \cap \mathbf{j}\right] \subseteq\left(\mathcal{N}_{\mathcal{A}} \cap \mathbf{j}\right)+\left(\mathcal{N}_{\mathcal{A}} \cap \mathbf{j}\right)=\mathcal{N}_{\mathcal{A}} \cap \mathbf{j} .
$$

Since $a \in \mathfrak{g}$ is arbitrary, this proves that $\mathcal{N}_{\mathcal{A}} \cap \mathfrak{j}$ is an ideal of $\mathfrak{g}$.

It remains to show that $\mathcal{N}_{\mathcal{A}} \cap \mathfrak{j} \subseteq \operatorname{rad} \mathcal{A}$. By Lemma 2.7, there exists an integer $m \geq 1$ such that the product of any $m$ elements in $\mathcal{N}_{\mathcal{A}} \cap \mathfrak{j}$ vanishes. 
On the other hand, as $\mathcal{N}_{\mathcal{A}} \cap \mathfrak{j}$ is an ideal of $\mathfrak{g}$, that is, $\left[\mathcal{N}_{\mathcal{A}} \cap \mathfrak{j}, \mathfrak{g}\right] \subseteq \mathfrak{g}$, we have $\left(\mathcal{N}_{\mathcal{A}} \cap \mathfrak{j}\right) \cdot \mathfrak{g} \subseteq \mathfrak{g} \cdot\left(\mathcal{N}_{\mathcal{A}} \cap \mathfrak{j}\right)+\left(\mathcal{N}_{\mathcal{A}} \cap \mathfrak{j}\right)$, hence the unital associative subalgebra $\mathcal{A}_{0}$ of $\mathcal{A}$ generated by $\mathfrak{g}$ satisfies

$$
\left(\mathcal{N}_{\mathcal{A}} \cap \mathfrak{j}\right) \cdot \mathcal{A}_{0}=\mathcal{A}_{0} \cdot\left(\mathcal{N}_{\mathcal{A}} \cap \mathfrak{j}\right)
$$

where for any subsets $S_{1}, \ldots, S_{k}$ of $\mathcal{A}$ we denote by $S_{1} \cdots S_{k}$ the linear subspace generated by all products $s_{1} \cdots s_{k}$ with $s_{1} \in S_{1}, \ldots, s_{k} \in S_{k}$. By iterating the above equality $m$ times we get

$$
\left(\mathcal{N}_{\mathcal{A}} \cap \mathfrak{j}\right) \cdot \mathcal{A}_{0} \cdots\left(\mathcal{N}_{\mathcal{A}} \cap \mathfrak{j}\right) \cdot \mathcal{A}_{0}=\left(\mathcal{N}_{\mathcal{A}} \cap \mathfrak{j}\right) \cdots\left(\mathcal{N}_{\mathcal{A}} \cap \mathfrak{j}\right) \cdot \mathcal{A}_{0} \cdots \mathcal{A}_{0}=\{0\} .
$$

In particular, for all $c \in \mathcal{N}_{\mathcal{A}} \cap \mathfrak{j}$ and $d \in \mathcal{A}_{0}$ we have $(c d)^{m}=0$. Since $\mathcal{A}_{0}$ is dense in $\mathcal{A}$ by hypothesis, the latter equality actually holds for all $d \in \mathcal{A}$ and implies that $\mathbf{1}-c d \in \mathcal{A}^{\times}$for all $d \in \mathcal{A}$. That is, $c \in \operatorname{rad} \mathcal{A}$ for arbitrary $c \in \mathcal{N}_{\mathcal{A}} \cap \mathfrak{j}$, and the proof is complete.

Now we can extend the main result of [Ti87] (or Theorem 1 in $\S 24$ of [BS01]) to FC-complete locally convex algebras with continuous inversion.

Theorem 4.3. Let $\mathcal{A}$ be a complex FC-complete CIA. Assume that $\mathfrak{g}$ is a complex Lie subalgebra of $\mathcal{A}$ and let $\mathcal{A}(\mathfrak{g})$ be the closed unital associative subalgebra of $\mathcal{A}$ generated by $\mathfrak{g}$. Then for every finite-dimensional solvable ideal $\mathfrak{j}$ of $\mathfrak{g}$ we have $[\mathfrak{j}, \mathfrak{g}] \subseteq \operatorname{rad}(\mathcal{A}(\mathfrak{g})) \subseteq \mathcal{Q}_{\mathcal{A}}$.

Proof. The inclusion $\operatorname{rad}(\mathcal{A}(\mathfrak{g})) \subseteq \mathcal{Q}_{\mathcal{A}(\mathfrak{g})}\left(\subseteq \mathcal{Q}_{\mathcal{A}}\right)$ follows at once from the definition of $\operatorname{rad}(\mathcal{A}(\mathfrak{g}))$.

To prove $[\mathfrak{j}, \mathfrak{g}] \subseteq \operatorname{rad}(\mathcal{A}(\mathfrak{g}))$, first note that according to Lemma 2.5(1), $\mathcal{A}(\mathfrak{g})$ is in turn a unital complex FC-complete CIA. Thus we may (and do) assume that $\mathcal{A}(\mathfrak{g})=\mathcal{A}$. Then define $\widetilde{\mathcal{A}}=\mathcal{A} / \operatorname{rad} \mathcal{A}$ and let $q: \mathcal{A} \rightarrow \widetilde{\mathcal{A}}$ the natural projection. We claim that $\widetilde{\mathcal{A}}^{\times}=q\left(\mathcal{A}^{\times}\right)$. The inclusion $q\left(\mathcal{A}^{\times}\right) \subseteq \widetilde{\mathcal{A}}^{\times}$ is trivial. For the converse, we assume that $q(a) \in \widetilde{\mathcal{A}}^{\times}$. Then there exists $b \in \mathcal{A}$ such that $a b, b a \in \mathbf{1}+\operatorname{rad} \mathcal{A} \subseteq \mathcal{A}^{\times}$. Hence $a$ is left and right invertible, which implies that $a \in \mathcal{A}^{\times}$. Now $\mathbf{1}+\operatorname{rad} \mathcal{A} \subseteq \mathcal{A}^{\times}$implies that

$$
q^{-1}\left(\widetilde{\mathcal{A}}^{\times}\right)=q^{-1}\left(q\left(\mathcal{A}^{\times}\right)\right)=\mathcal{A}^{\times}+\operatorname{rad} \mathcal{A}=\mathcal{A}^{\times},
$$

and this entails that $\sigma_{\widetilde{\mathcal{A}}}(q(a))=\sigma_{\mathcal{A}}(a)$ for each $a \in A$. Then Remark 2.3 and Lemma 2.5(3) show that $\widetilde{\mathcal{A}}$ is a unital complex FC-complete CIA with $\operatorname{rad} \widetilde{\mathcal{A}}=\{0\}$. We are going to apply Lemma 4.1 to the ideal $q(\mathfrak{j})$ of the Lie subalgebra $q(\mathfrak{g})$ of $\widetilde{\mathcal{A}}$.

In fact, let $a \in q(\mathfrak{g})$ and $b \in q(\mathfrak{j})$ be such that $\left(\operatorname{ad}_{q(\mathfrak{g})} a\right) b=\lambda b$ for some $\lambda \in \mathbb{C} \backslash\{0\}$. Then $b \in \mathcal{N}_{\widetilde{\mathcal{A}}} \cap q(\mathbf{j}) \subseteq \operatorname{rad} \widetilde{\mathcal{A}}=\{0\}$ by Corollary 3.4 and Proposition 4.2. Consequently, the linear mapping $\left.\left(\operatorname{ad}_{q(\mathfrak{g})} a\right)\right|_{q(\mathfrak{j})}: q(\mathfrak{j}) \rightarrow q(\mathfrak{j})$ has no non-zero eigenvalue, and thus it is nilpotent. It then follows from Lemma 4.1 that $[q(\mathfrak{g}), q(\mathfrak{j})]=\{0\}$, that is, $[\mathfrak{g}, \mathfrak{j}] \subseteq \operatorname{Ker} q=\operatorname{rad} \mathcal{A}$. 
Corollary 4.4. Let $\mathcal{A}$ be a complex FC-complete CIA. Assume that $\mathfrak{g}$ is a finite-dimensional complex Lie subalgebra of $\mathcal{A}, \mathfrak{r}$ its solvable radical, and $\mathcal{A}(\mathfrak{g})$ the closed unital associative subalgebra of $\mathcal{A}$ generated by $\mathfrak{g}$. Then $[\mathfrak{g}, \mathfrak{r}] \subseteq \operatorname{rad}(\mathcal{A}(\mathfrak{g})) \subseteq \mathcal{Q}_{\mathcal{A}}$

\section{CIA-linear Lie groups}

Definition 5.1. A finite-dimensional Lie group $G$ is said to be linear if it is isomorphic to a (closed) Lie subgroup of some $\mathrm{GL}_{n}(\mathbb{R})$. Obviously, this condition is equivalent to the requirement that $G$ is isomorphic to a Lie subgroup of some finite-dimensional unital associative algebra $\mathcal{A}$.

We call $G$ CIA-linear if there exists an injective continuous homomorphism $\eta: G \rightarrow \mathcal{A}^{\times}$for some FC-complete CIA $\mathcal{A}$.

Lemma 5.2. Let $\mathcal{A}$ be a complex FC-complete CIA and $x \in \mathcal{A}$ quasinilpotent such that $e^{\mathbb{R} x}$ is relatively compact in $\mathcal{A}^{\times}$. Then $x=0$.

Proof. Let $\mathcal{A}_{0} \subseteq \mathcal{A}$ be a maximal commutative subalgebra containing $x$. Then $\mathcal{A}_{0}$ is an FC-complete commutative CIA with $\{0\}=\sigma_{\mathcal{A}}(x)=\sigma_{\mathcal{A}_{0}}(x)$ (Lemma 2.5). We may therefore assume that $\mathcal{A}$ is commutative.

Then the Gelfand transform $\mathcal{G}: \mathcal{A} \rightarrow C(\widehat{\mathcal{A}}), a \mapsto \widehat{a}$, satisfies $\|\widehat{a}\|_{\infty}=$ $r_{\mathcal{A}}(a)$, so that $\operatorname{rad} \mathcal{A}=\mathcal{Q}_{\mathcal{A}}=\operatorname{ker} \mathcal{G}$ is a closed 2-sided ideal of $\mathcal{A}$. We conclude that the closure $K \subseteq \mathcal{A}^{\times}$of $e^{\mathbb{R} x}$ is contained in the closed affine subspace $U:=\mathbf{1}+\operatorname{rad} \mathcal{A}=\mathcal{G}^{-1}(\mathbf{1})$, which is contained in $\mathcal{A}^{\times}$, hence $K$ is a subgroup of $\mathcal{A}^{\times}$.

Next we observe that the spectral mapping theorem implies that

$$
\operatorname{Exp}:\left(\mathcal{Q}_{\mathcal{A}},+\right)=(\operatorname{rad}(\mathcal{A}),+) \rightarrow U, \quad x \mapsto e^{x},
$$

is a diffeomorphism with inverse the logarithm function $\log : U \rightarrow \operatorname{rad}(\mathcal{A})$, which in turn is given by a convergent power series. We conclude that $\log (K)$ is a compact subgroup of the locally convex space $(\operatorname{rad}(\mathcal{A}),+)$, hence trivial, and this implies that $x=0$.

The main result of our paper is the following theorem:

TheOREM 5.3. For a connected finite-dimensional Lie group $G$ the following are equivalent:

(1) $G$ is CIA-linear.

(2) The continuous homomorphisms $\eta: G \rightarrow \mathcal{A}^{\times}$into the unit groups of $F C$-complete CIAs separate the points of $G$.

(3) $G$ is linear.

Proof. $(1) \Rightarrow(2)$ and $(3) \Rightarrow(1)$ hold trivially. Therefore it remains to show that (2) implies (3).

Assume that $G$ satisfies (2). Let $\mathfrak{g}:=\mathbf{L}(G)$ be the Lie algebra of $G$ and $\mathfrak{g}=\mathfrak{r} \rtimes \mathfrak{s}$ be a Levi decomposition. 
STEP 1. For $0 \neq x \in[\mathfrak{r}, \mathfrak{g}]$ the subgroup $\exp _{G}(\mathbb{R} x)$ is not relatively compact.

We argue by contradiction. Let $0 \neq x \in[\mathfrak{r}, \mathfrak{g}]$. From (2) and the fact that $\mathfrak{g}$ is finite-dimensional it follows that there exists a complex FC-complete CIA $\mathcal{A}$ and a morphism of Lie groups $\eta: G \rightarrow \mathcal{A}^{\times}$for which $\mathbf{L}(\eta): \mathfrak{g} \rightarrow \mathcal{A}$ is injective. Now Corollary 4.4 implies that $\mathbf{L}(\eta)([\mathfrak{g}, \mathfrak{r}]) \subseteq \mathcal{Q}_{\mathcal{A}}$, so that Lemma 5.2 entails that $\eta\left(\exp _{G}(\mathbb{R} x)\right)=e^{\mathbb{R} \mathbf{L}(\eta) x}$ is not relatively compact in $\mathcal{A}^{\times}$, and this implies that $\exp _{G}(\mathbb{R} x)$ cannot be relatively compact in $G$.

Step 2. Let $R:=\left\langle\exp _{G} \mathfrak{r}\right\rangle$ denote the radical of $G$. Then $R$ is a linear Lie group.

Let $R^{\prime}$ be the commutator subgroup of $R$. Since $\mathbf{L}\left(R^{\prime}\right)=[\mathfrak{r}, \mathfrak{r}]$, Step 1 implies that $\exp _{G}(\mathbb{R} x)$ is not relatively compact in $G$ for $0 \neq x \in[\mathfrak{r}, \mathfrak{r}]$. This implies that $R^{\prime}$ is closed ([Ho65, XVI.2.3/4]) and does not contain circle groups. Hence it is a simply connected nilpotent Lie group, so that all compact subgroups of $R^{\prime}$ are trivial. Now [Ho65, XVIII.3.2] implies that $R$ is a linear Lie group.

SteP 3. The Levi subgroup $S:=\left\langle\exp _{G} \mathfrak{s}\right\rangle \leq G$ is linear.

Let $q_{S}: \widetilde{S} \rightarrow S$ denote the universal covering and $\eta_{S}: \widetilde{S} \rightarrow \widetilde{S}_{\mathbb{C}}$ be the universal complexification. Then $S$ is linear if and only if $S$ is a quotient of $\eta_{S}(\widetilde{S})$, i.e., $\operatorname{ker} \eta_{S} \supseteq \operatorname{ker} q_{S}$ ([Ho65, XVII.3.3]). Since $\mathcal{A}$ is a complex FCcomplete CIA, the homomorphism of Lie algebras $\mathbf{L}(\eta): \mathfrak{s} \rightarrow \mathcal{A}$ extends to a homomorphism $\mathbf{L}(\eta)_{\mathbb{C}}: \mathfrak{s}_{\mathbb{C}} \rightarrow \mathcal{A}$, which in turn integrates to a morphism of Lie groups $\eta_{\mathbb{C}}: \widetilde{S}_{\mathbb{C}} \rightarrow \mathcal{A}^{\times}$with $\mathbf{L}\left(\eta_{\mathbb{C}}\right)=\mathbf{L}(\eta)_{\mathbb{C}}$. Thus $\left.\mathbf{L}\left(\eta_{\mathbb{C}} \circ \eta_{S}\right)\right|_{\mathfrak{s}}=$ $\left.\mathbf{L}(\eta)\right|_{\mathfrak{s}}$, and hence $\eta_{\mathbb{C}} \circ \eta_{S}=\eta \circ q_{S}$, so that ker $\eta_{S} \subseteq q_{S}^{-1}(\operatorname{ker} \eta)$. By (2), the homomorphisms $\eta: G \rightarrow \mathcal{A}^{\times}$separate the points of $S$, and we conclude that ker $\eta_{S} \subseteq \operatorname{ker} q_{S}$, showing that $S$ is linear.

SteP 4. $G$ is linear because $R$ and $S$ are linear ([Ho65, XVIII.4.2]).

Acknowledgments. The first-named author was partially supported by grant CERES 4-187/2004.

\section{References}

[Al65] G. R. Allan, A spectral theory for locally convex algebras, Proc. London Math. Soc. (3) 15 (1965), 399-421.

[Bel06] D. Beltiţă, Smooth Homogeneous Structures in Operator Theory, Chapman \& Hall/CRC Monogr. Surveys Pure Appl. Math. 137, Chapman \& Hall/CRC Press, Boca Raton, 2006.

[BS01] D. Beltiţă and M. Şabac, Lie Algebras of Bounded Operators, Oper. Theory Adv. Appl., 120, Birkhäuser, Basel, 2001. 
[Bi07] H. Biller, Analyticity and naturality of the multi-variable functional calculus, Expo. Math. 25 (2007), 131-163.

[Co68] I. Colojoară, Elements of Spectral Theory, Ed. Acad., Bucureşti, 1968 (in Romanian).

[G102] H. Glöckner, Algebras whose groups of units are Lie groups, Studia Math. 153 (2002), 147-177.

[GN06] H. Glöckner and K.-H. Neeb, Infinite-Dimensional Lie Groups, book in preparation, Springer, to appear.

[GL73] D. L. Gurariĭ and Yu. I. Lyubich, An infinite-dimensional analogue of Lie's theorem on weight, Funktsional. Anal. i Prilozhen. 7 (1973), no. 1, 41-44 (in Russian).

[He93] A. Ya. Helemskii, Banach and Locally Convex Algebras, Oxford Univ. Press, New York, 1993.

[Ho65] G. Hochschild, The Structure of Lie Groups, Holden Day, San Francisco, 1965.

[KOO98] A. El Kinani, L. Oubbi and M. Oudadess, Spectral and boundedness radii in locally convex algebras, Georgian Math. J. 5 (1998), 233-241.

[Koe69] G. Köthe, Topological Vector Spaces I, Grundlehren Math. Wiss. 159, Springer, Berlin, 1969.

[LV94] D. Luminet and A. Valette, Faithful uniformly continuous representations of Lie groups, J. London Math. Soc. (2) 49 (1994), 100-108.

[Mil83] J. Milnor, Remarks on infinite-dimensional Lie groups, in: B. DeWitt and R. Stora (eds.), Relativity, Groups and Topology, II (Les Houches, 1983), North-Holland, Amsterdam, 1984, 1007-1057.

[Ne06] K.-H. Neeb, Towards a Lie theory of locally convex groups, Japanese J. Math. 1 (2006), 291-468.

[Ro56] M. Rosenblum, On the operator equation $B X-X A=Q$, Duke Math. J. 23 (1956), 263-269.

[Sa96] M. Şabac, Nilpotent elements and solvable actions, Collect. Math. 47 (1996), 91-104.

[Ti87] Yu. V. Turovskiü, Commutativity modulo the Jacobson radical of the associative envelope of a Lie algebra, in: Spectral Theory of Operators and Its Applications, No. 8, Elm, Baku, 1987, 199-211 (in Russian).

[Wae67] L. Waelbroeck, Théorie des Algèbres de Banach et des Algèbres Localement Convexes, 2nd ed., Sém. Math. Sup. 2, Presses Univ. de Montréal, Montréal, Qué., 1967.

Institute of Mathematics "Simion Stoilow" of the Romanian Academy

P.O. Box 1-764

Bucureşti, Romania

E-mail: Daniel.Beltita@imar.ro
Department of Mathematics Darmstadt University of Technology Schlossgartenstrasse 7 D-64289 Darmstadt, Germany E-mail: neeb@mathematik.tu-darmstadt.de

Received August 23, 2006

Revised version January 22, 2008 